\title{
Reconstruction of image sequences from ungated and scanning-aberrated laser scanning microscopy images of the beating heart
}

\author{
Olivia Mariani, Student Member, IEEE, Alexander Ernst, Nadia Mercader, and Michael Liebling, Member, IEEE
}

\begin{abstract}
Fluorescence laser-scanning microscopy is a wellestablished imaging technique in biology, available in many imaging facilities to investigate structures within live animal embryos such as zebrafish. Laser scanning microscopes (LSM) are limited when used to study dynamic heart morphology or function. Despite their ability to resolve static cardiac structures, the fast motion of the beating heart introduces severe artifacts in the scanned images and gating the acquisitions to the heartbeat is difficult to implement on traditional microscopes. Furthermore, although alternative high-speed imaging instruments exist, they are not widely available (due to cost or hardware complications), putting dynamic cardio-vascular imaging off-limits for many researchers. Here, we propose a method that allows imaging the beating heart on conventional LSMs. Our approach takes a set of images containing scanning aberrations, each triggered at an arbitrary time in the cardiac cycle, and assembles an image sequence that covers a single cardiac heartbeat. The steps are: (i) frame sorting by solving a traveling salesman problem; (ii) heartbeat duration estimation; and (iii) scan-delay compensation via space-time resampling. We characterize the performance of our method on synthetic data under several light intensities and scanning speeds. We further illustrate our method's applicability on experimental images acquired in live zebrafish larvae, and show that the reconstruction quality approaches that of fast, state-of-the-art microscopes. Our technique opens the possibility of using LSMs to carry out studies of cardiac dynamics, without the need for prospective gating or fast microscopes.
\end{abstract}

Index Terms-Computational microscopy, laser scanning microscopy, confocal microscopy, fast microscopy, scanning aberration compensation, combinatorial optimization, traveling salesman problem, image and video sampling, cardiac imaging, zebrafish imaging,

\section{INTRODUCTION}

In vivo microscopy is an essential tool to study organ development at the cellular level in embryos of animal models. In zebrafish, whose embryos and larvae are mostly transparent, most organs can be directly viewed under a light microscope. In particular, it is possible to observe the developing zebrafish

O. Mariani is with the Computational Bioimaging Group, Idiap reasearch institute, Martigny, VS, CH-1920, Switzerland, and the Swiss Federal Institue of Technology Lausanne, Lausanne, VD, CH-1024, Switzerland e-mail: olivia.mariani@idiap.ch

A. Ernst and N. Mercader are with the Institute of Anatomy, University of Bern, CH-3000 Bern, Switzerland.

M. Liebling is with the Computational Bioimaging Group, Idiap reasearch institute, Martigny, VS, CH-1920, Switzerland and the Electrical \& Computer Engineering, University of California, Santa Barbara, CA 93106, USA

This paper has supplementary downloadable material available at http://ieeexplore.ieee.org, provided by the authors. The material includes ten multimedia MP4 format movie clips showing the main steps described in the paper, from data acquisition to validation and reconstruction, with synthetic and experimental data. This material is $32.8 \mathrm{MB}$ in size. heart, whose early developmental stages are very similar to the ones in humans. Nevertheless, high-resolution and depthresolved cardiac imaging are hampered by the rapid beating of the heart. This limitation is especially important when acquiring images with a laser-scanning microscope (LSM; e.g. a confocal LSM, two-photon LSM, etc.). Laser scanning microscopy techniques, which allow optical sectioning, are widely used because of the many advantages they offer. These include high spatial resolution, possibility of acquiring images in arbitrary regions of interest, common availability of LSMs in imaging centers, and existence of many established and LSM-compatible sample mounting protocols. The limitation of LSMs, when imaging the heart, is the scanning procedure, which has contradicting requirements. The speed should ideally be high enough to avoid compromising the temporal resolution of heartbeats, yet slow enough to collect dim light and achieve acceptable signal-to-noise ratio (SNR). Naive imaging of the zebrafish heart leads to aberrations of two forms: first, the slow scanning speed creates scanning aberrations because the heartbeats during the acquisition of a single image (Fig. 1(a)); second, the variable time spent in between frames (to write the data) leads to images captured at arbitrary phases of the cardiac cycle (Fig. 2(a)). There are various ways to avoid scanning artifacts when imaging a fastmoving sample but none of them offers simultaneously the molecular insights possible with fluorescence microscopy, high enough spatial and temporal resolution, compatibility with a wide range of sample mounting protocols, and availability in most core imaging facilities. For example, the scan speed could be increased, such as demonstrated in optical coherence tomography (OCT) [1], where heart morphology and function in non-transparent embryos (both in avians [2], [3] and mammals [4]) is possible. However, in OCT, the scan speed is primarily limited by the instrument (mirror scan speed and light source intensity) whereas in fluorescence imaging, it is the number of available fluorophores that can emit light, which is the limiting factor, and hence faster scanning severely affects the SNR. For example, fluorescence LSMs equipped with a resonant scanner [5] can offer sufficient temporal and spatial resolution to image fast cells [6] but the faster scanning speed requires that the samples be very bright and that emitted photon losses be minimized as the pixel dwell time (the time spent by the scanner on a single point) is lower. High-performance point scanning confocal microscopy systems that combine highspeed scanners and light-efficient collection approaches [7] are sometimes available to researchers but they remain costly. 

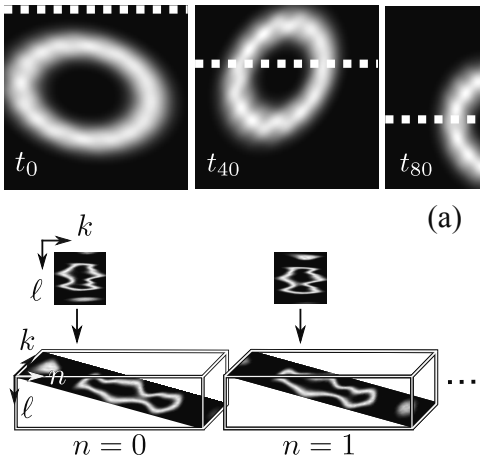

(b)

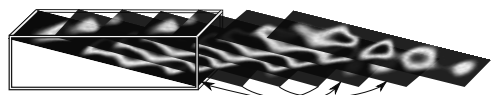

(c)

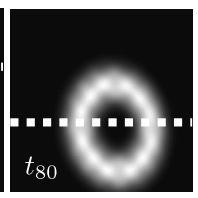

a)

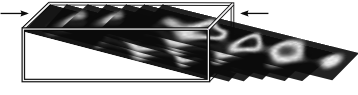

(d)

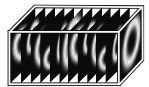

(e)
Fig. 1. Summary of acquisition and proposed reconstruction procedure. (a) Artifacts arise when a fast-moving object is imaged via slow scanning (one line at a time). Each row in the resulting frame (right) comes from a different cardiac contraction phase. (b)-(e) Our reconstruction procedure consists of: (b) collect multiple aberrated frames, which can be represented as slanted planes in space-time; (c) sort the frames according to increasing cardiac phase; (d) estimate the heartbeat period and produce a dense cardiac cycle; (e) resample the data on a uniform space-time grid that covers one heartbeat. See also Supplementary Movie 1 for Steps (a) and (b).

Line scanning confocal microscopes [8], [9] and spinning disk microscopes [10], [11] allow imaging at high speeds yet at the cost of less regional scanning flexibility and crosstalk in thicker samples compared to point scanning systems. Light sheet microscopy [12]-[15] satisfies the spatial and temporal resolution requirements for cardiac imaging but is not always available, in particular at imaging centers that have only few instruments; since the cost of purchasing a reliable turnkey microscope is high (ranging from tens to hundreds of thousands of USD or Euros), LSMs are a common first choice as they cover the needs and applications of many users (Table I). Indeed, LSMs offer exquisite sensitivity and spatial resolution, optical sectioning capabilities in a wide range of fluorescent samples, possibility to scan only small regions of interest (ROI), compatibility with a variety of mounting techniques, and widespread options for both single and multi-photon excitation. While purchasing or building a new microscope dedicated specifically to a cardiac imaging project (e.g. a light-sheet microscope) offers the best quality images at high speed, it will only be a sensible investment for researchers who image the heart regularly and can dedicate major funds to purchasing and maintaining such a microscope, or who have the skills to assemble a more cost effective version on their own [16], [17]. If LSM images of the heart were not severely corrupted by scanning artifacts, the possibility of using already existing (LSM) resources at a core facility would therefore be a practical, versatile, and economical alternative.

In this paper, we consider the problem of enabling such an alternative. Specifically, we aim at developing a computational method that takes a set of scanning-aberrated LSM images of the beating heart as input (as obtained, e.g., by use of an unmodified, off-the-shelf commercial LSM) and returns an image sequence (movie) with a faithful representation of the beating heart geometry covering one heartbeat (Fig. 1). Although, challenges of imaging the heart on slow devices have been considered in the past, we are not aware of methods that directly address the problem at hand.

\section{A. Related work}

Cardiac phase sorting methods have been considered in the past. Zhang and Pless [19] sorted cardiopulmonary MRI images according to the cardiac and respiratory phases by projecting the images on a low-dimensional manifold [20], and used level sets to segment the images. Kellman et al. [21] rebinned MRI images according to cardiac phase, with the phase determined with ECG timing data. In free-breathing MRI, Feng et al. [22] extracted the motion signal from different coils to extract estimations of the cardiac and respiratory frequencies, and then sort the acquired data according to the cardiac and respiratory phases. In OCT, Liu et al. [3] pooled OCT images from multiple sequences of continuous and undistorted heartbeats to obtain higher framerate sequences by determining their relative phases. Similarly, Happel et al. [23] increased the frame rate in OCT using the string-length method [24], [25]. Approaches based on the string-length method assume that data are collected in a strictly sequential fashion and with known timestamps, which we do not assume here. Tralie and Berger [26] created high temporal resolution movies of periodic subjects by estimating the period of the signal then the phases within a sliding window. We recently proposed a cardiac phase sorting method (based on a traveling salesman problem (TSP) formulation) to recover the cardiac phase of a series of still images of a beating heart [27]. A similar TSP approach, albeit using different distance metrics and boundary conditions, has been proposed by Hanslovsky et al. [28] to sort out-of-order electron microscopy image stacks. Despite the ability of the above cardiac imaging methods to increase the framerate given instantaneous snapshots, none of them tackles the problem of compensating for aberrations introduced by scanning. Methods that compensate for scanning aberrations have been developed separately in other contexts, including for de-interlacing in television [29]. In the field of microscopy, Surgon et al. [30] have proposed a method to reconstruct a single frame without aberrations given a series of un-gated images. Nevertheless, this method falls short of reconstructing entire sequences, which is necessary to study cardiac dynamics.

\section{B. Contributions}

In this paper we present a fully computational method to recover cardiac heartbeat series from scanning-aberrated snapshots, with only minimal knowledge of the imaging device characteristics and without the need for additional hardware or hardware modifications, making our method well-suited for post-processing images acquired on a wide range of LSMs, even from devices that do not allow low-level acquisition adjustments by the user (a common limitation of commercial LSMs). We formulate the image reconstruction problem as a combined sorting and space-time unshearing problem, whose 
TABLE I

COMPARISON OF OPTICAL SECTIONING-CAPABLE MICROSCOPY METHODS (CONVENTIONAL LASER SCANNING MICROSCOPY (LSM), FAST LSM (AIRYSCAN [18]), LIGHT-SHEET MICROSCOPY, AND LSM COMBINED WITH OUR PROPOSED RECONSTRUCTION METHOD). MORE STARS (*) INDICATE PREFERRED METHOD. OUR PROPOSED APPROACH EXTENDS THE USE RANGE OF WIDELY AVAILABLE LSMS TO CARDIAC IMAGING.

\begin{tabular}{|c|c|c|c|c|c|c|}
\hline & $\begin{array}{c}\text { Accuracy of cardiac } \\
\text { shape in images }\end{array}$ & $\begin{array}{l}\text { Sample preparation } \\
\text { and mounting }\end{array}$ & $\begin{array}{c}\text { Flexible ROI } \\
\text { Imaging }\end{array}$ & Multi-photon options & Cost & Availability \\
\hline LSM & $*$ & Standard & $* * * *$ & $* * * *$ & $\$ \$ \$$ & $* * * *$ \\
\hline Fast LSM & $* * * *$ & Standard & $* * * *$ & $* *$ & $\$ \$ \$ \$$ & $* *$ \\
\hline Light Sheet & $* * * *$ & Non-standard & $*$ & $* *$ & $\$ \$ \$ \$ / \${ }^{a}$ & $* *$ \\
\hline LSM + our method & $* *$ to $* * * * b$ & Standard & $* * * *$ & $* * * *$ & $\$ \$ \$$ & $* * * *$ \\
\hline
\end{tabular}

${ }^{a}$ Commercial light-sheet microscopes are very expensive, but do-it-yourself options [16], [17] can be more affordable if skills and time are available.

${ }^{b}$ Dependent on cardiac phase and cell velocity

free parameters are unknown (cardiac phase, timestamps, or cardiac heart-beat frequency).

We propose to solve this image reconstruction problem by splitting it into two variational problems to recover the unknown parameters, followed by data resampling. Specifically, we cast the problem of retrieving the images' unknown phases as a shortest path search problem with periodic boundary conditions to match the cyclical nature of the cardiac heartbeat and with a pixel-wise similarity criterion as a distance metric, an approach whose efficacy we already demonstrated for sorting unaberrated cardiac images [27]. Following sorting we formulate the second problem as a spacetime shearing problem whose unknown spacing parameter we recognize to coincide with the unknown cardiac period. Again, we use a variational approach to determine the unknown parameter, based on a minimum image difference criterion between adjacent, resampled slices. . In addition to detailing the method, we characterize the algorithm on synthetic data, demonstrate its applicability on experimental microscopy data, and qualitatively compare the reconstruction to experimental data acquired with two highspeed modalities.

Given the novelty of the reconstruction problem and method, we have characterized and validated our proposed approach using different datasets and settings. First, we reconstructed synthetically generated data (with and without noise corruption) for which the ground-truth signal was known, then quantitated the reconstruction accuracy as a function of the main free parameters. Next we carried out reconstructions on experimentally acquired images of the beating heart in zebrafish larvae, acquired on a (slow) confocal LSM, and compared our reconstructed images to those obtained via direct fast imaging methods (fast confocal LSM and lightsheet microscopy), suggesting that our method can reliably reconstruct the geometry of the beating heart (with some limitations).

The paper is organized as follows. In Section II, we provide a formal description of the imaging model and problem. In Section III, we propose a sorting solution based on a TSP solver and a compensation for scanning artifacts. In Section IV, we evaluate our method on synthetic and experimental data. In Section V, we discuss our findings. Finally, we conclude in Section VI.

\section{Model AND PRoblem DeFinition}

We consider a time-varying two-dimensional image whose intensity at spatial location $(x, y)$ and time $t$ we denote by $f(x, y, t)$. The image intensity varies periodically with a period $T$, such that, for any $t \in \mathbb{R}$, we have:

$$
f(x, y, t)=f(x, y, t+T) .
$$

We further define a uniformly-sampled image series $\mathbf{f}$ that covers the duration $T$ of one heartbeat:

$$
\mathbf{f}[k, \ell, n]=f\left(k \Delta_{x}, \ell \Delta_{y}, n \Delta_{t}\right),
$$

where $\Delta_{x}$ and $\Delta_{y}$ are the pixel width and height, respectively, $k=0, \ldots, K-1$ and $\ell=0, \ldots, L-1$ are the row and column indices, respectively, $n=0, \ldots, N-1$ denotes the time frame index, and $\Delta_{t}=\frac{T}{N}$ denotes the time interval between frames.

We denote by $\mathbf{g}[k, \ell, n], k=0, \ldots, K-1, \ell=0, \ldots, L-1$, and $n=0, \ldots, N-1$, the discrete image series measured by the scanning microscope, which we model by:

$$
\mathbf{g}[k, \ell, n]=f\left(k \Delta_{x}, \ell \Delta_{y}, t_{n}+s_{\ell} \ell\right),
$$

where $s_{\ell}$ is the vertical scanning rate (in units of time per row, where we assume that the lateral scanning, which is fast compared to the vertical scanning rate, is instantaneous) and the $t_{n}$ 's are the times at which the corresponding frames $\mathbf{g}[$ : $,:, n]$ are triggered. These starting times occur at increasing, but arbitrarily-spaced times:

$$
t_{0}<\ldots<t_{n}<\ldots<t_{N-1}, \quad t_{n} \in \mathbb{R} .
$$

Given the above definitions, the image reconstruction problem is to obtain an estimate $\tilde{\mathbf{f}}$ of the uniformly sampled image sequence $\mathbf{f}$ given the sheared measurements $\mathbf{g}$ and knowledge of the microscope's vertical scan speed $s_{\ell}$ (but without knowledge of the underlying heartbeat period $T$ nor the starting times $t_{n}$ ). 


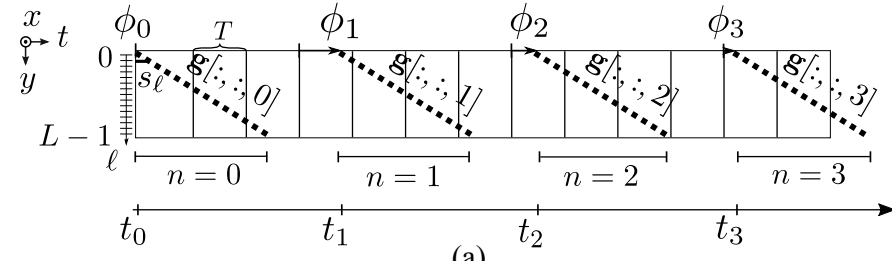

(a)

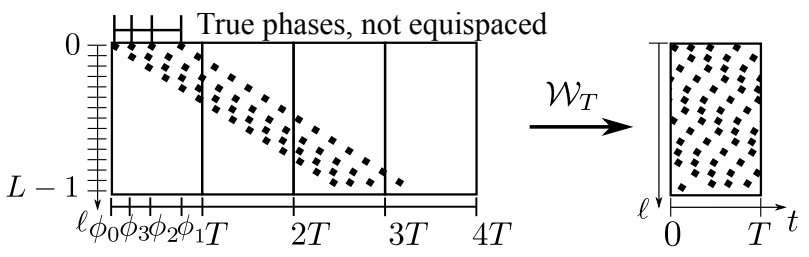

(b)

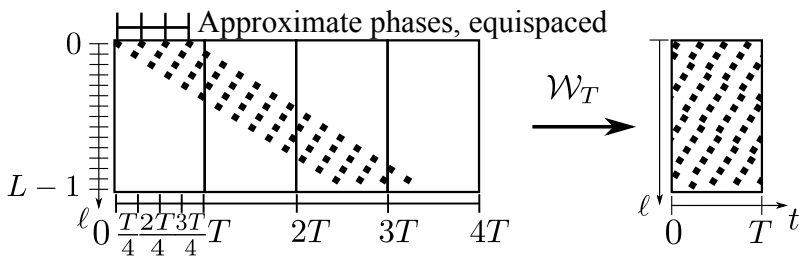

(c)

Fig. 2. Sampling model for scanning, true and approximated frame phase distribution. (a) The scanning acquisition results in sheared images $\mathbf{g}[k, \ell, n]$, (diagonal dots, one image line corresponds to one dot), which can span multiple periods $T$ of the original signal. Acquisition is triggered at arbitrary times $t_{n}$ with corresponding phases $\phi_{n}$. (b) Frames sorted according to their true (but unknown) cardiac phase. Wrapping the data to a single period results in a dense sampling of the space-time for one heart-beat. Note that given the stochastic nature of the start times $t_{n}$, the phases are not evenly distributed over the heartbeat. (c) While our sorting procedure produces reliable sorting, it does not provide the phase positions, which we approximate as equispaced. We investigate the error introduced by this approximation in Fig. 4(a).

\section{Phase SORTING, PERIOd COMPLETION AND ESTIMATION METHOD}

To reconstruct a uniformly sampled image series of the beating heart over one period from the scanning-aberrated data that we measure, we proceed in three steps, which can be summarized as follows: first, we sort the scanning-aberrated frames $\mathrm{g}$ (Fig. 1(a) and (b)) so that the starting times $t_{n}$ are in order of increasing cardiac phase (see Section III-A, Fig 1(c)); next, we estimate the duration $T$ of one heartbeat (see Section III-B, Fig 1(d)) such that the pixels in the sorted frames can then be assigned to their position in the cardiac phase space; finally, we resample the phase-ordered scanning-aberrated data on a uniform grid to obtain the final estimate $\tilde{\mathbf{f}}$ (see Section III-C, Fig 1(e)). Note that, in [27], only the special case where the vertical scanning rate is instantaneous was considered, i.e. $s_{\ell}=0$, and the problem therefore limited to sorting frames.

\section{A. Sorting scanning-aberrated images according to cardiac phase}

We follow the phase-sorting method described in [27], and define the cardiac phases $\phi_{n}$ of the $n^{\text {th }}$ frame $\mathbf{g}[:,:, n]$, as the wrapping operation $\phi_{n}=\mathcal{W}_{T}\left(t_{n}\right)=t_{n}+m T, m \in \mathbb{Z}$, s.t $\phi_{n} \in[0, T)$. The task of sorting the frames is equivalent to finding a permutation $\sigma:\{0, \ldots, N-1\} \rightarrow\{0, \ldots, N-1\}$, $m \mapsto n=\sigma(m)$ such that the phases $\phi_{\sigma(0)}, \ldots, \phi_{\sigma(N-1)}$ are in increasing order:

$$
\phi_{\sigma(0)} \leq \cdots \leq \phi_{\sigma(N-1)} .
$$

Given a sequence of images $\mathbf{g}$, we denote by $\mathbf{g}^{\sigma}$ the phaseordered (but still scan-sheared) frame sequence obtained by applying the permutation $\sigma$ to $\mathrm{g}$ :

$$
\mathbf{g}^{\sigma}[:,:, m]=\left.\mathbf{g}[:,:, n]\right|_{n=\sigma(m)}, \quad m=0, \ldots, N-1 .
$$

We assume the cardiac cycle to be a closed repeating cycle and the images corresponding to adjacent phases in the cycle to be similar. We further assume that each cardiac phase produces a unique image (symmetrical patterns are excluded). We formulate the phase-ordering as a minimization problem, with image difference as a similarity criterion, so that this problem boils down to a classical combinatorial optimization problem. To do so, we define the cost of a given candidate permutation $\sigma^{\prime}$ (with $\sigma^{\prime}\left(t_{0}\right)=0$ ) as:

$$
\begin{array}{r}
C\left(\mathbf{g}, \sigma^{\prime}\right)=\sum_{m=0}^{N-2} d\left(\mathbf{g}^{\sigma^{\prime}}[:,:, m], \mathbf{g}^{\sigma^{\prime}}[:,:, m+1]\right) \\
+d\left(\mathbf{g}^{\sigma^{\prime}}[:,:, N-1], \mathbf{g}[:,:, 0]\right),
\end{array}
$$

where the frame-wise distance operator $d(\cdot, \cdot)$ between two 2D frames $\mathbf{a}$ and $\mathbf{b}$ is defined as:

$$
d(\mathbf{a}, \mathbf{b})=\sum_{k=0}^{K-1} \sum_{\ell=0}^{L-1}|\mathbf{a}[k, \ell]-\mathbf{b}[k, \ell]| .
$$

Given that the distance is symmetrical, permutations that lead to a minimal cost come in pairs:

$$
\left\{\tilde{\sigma}^{T}, \tilde{\sigma}\right\}=\underset{\sigma^{\prime} \in S_{N-1}}{\arg \min } C\left(\mathbf{g}, \sigma^{\prime}\right)
$$

where $\tilde{\sigma}$ is the ordering that satisfies Eq. (5), and $\tilde{\sigma}^{T}$ the same permutation in reverse order.

Given an $N \times N$ (symmetrical) table $\mathbf{D}$ whose entries $D_{j, k}=d(\mathbf{g}[:,:, j], \mathbf{g}[:,:, k])$ contain the frame-wise distances between all frame pairs, we can recognize that Problem (9) is an incarnation of the TSP: the permutations we seek correspond to finding a path that visits each image (and comes back to the starting image) while minimizing the distance traveled between adjacent images.

In order to solve this problem efficiently and robustly, we spatially-average and downsample each frame $\mathbf{g}[:,:, n]$, $n=0, \ldots, N-1$ before computing the distance table $\mathbf{D}$. Since there are $(N-1)$ ! possible frame permutations $(N$ is the number of frames and the first frame is fixed) exploring all combinations would be prohibitively expensive. Instead, we use the TSP solver package Concorde [31] with the linear programming solver QSopt [32]. The computational time and complexity of the Concorde solver was discussed in [33], and shown to follow a function of the form $a \cdot b^{\sqrt{N}}$, with $a=0.21$ and $b=1.24194$ [34], which is still exponential, but grows much slower than $(N-1)$ !. We solve the problem of choosing between $\tilde{\sigma}$ and the reverse permutation $\tilde{\sigma}^{\top}$ in the next step. 


\section{B. Estimation of the heartbeat period}

Although the previous step produces a sorted series of images, the scanning artifacts remain. In order to compensate for them, we estimate the heartbeat period, which specifies the spacing of frames in space-time. Concretely, the phase-ordered images, $\mathbf{g}^{\sigma}$, are related to the underlying continuous signal $f$ via:

$$
\begin{aligned}
\mathbf{g}^{\tilde{\sigma}}[k, \ell, m] & =f\left(k \Delta x, \ell \Delta y, t_{\tilde{\sigma}(m)}+s_{\ell} \ell\right) \\
& =f\left(k \Delta x, \ell \Delta y, \mathcal{W}_{T}\left(t_{\tilde{\sigma}(m)}+s_{\ell} \ell\right)\right) \\
& =f\left(k \Delta x, \ell \Delta y, \mathcal{W}_{T}\left(\mathcal{W}_{T}\left(t_{\tilde{\sigma}(m)}\right)+s_{\ell} \ell\right)\right)
\end{aligned}
$$

where Equation (11) results from the periodicity of $f$ and Equation (12) from a property of the wrapping operation. In Equation (12), we recognize the phase $\tilde{\phi}_{m}=\mathcal{W}_{T}\left(t_{\tilde{\sigma}(m)}\right)$ of the $m$ th frame of the ordered stack, which we substitute therein to obtain:

$$
\mathrm{g}^{\tilde{\sigma}}[k, \ell, m]=f\left(k \Delta x, \ell \Delta y, \mathcal{W}_{T}\left(\tilde{\phi}_{m}+s_{\ell} \ell\right)\right) .
$$

We next approximate the (unknown) phase as:

$$
\tilde{\phi}_{m} \approx \frac{T m}{N} \text {. }
$$

This approximation, illustrated in Fig. 2, leads to the following expression:

$$
\mathrm{g}^{\tilde{\sigma}}[k, \ell, m] \approx f\left(k \Delta x, \ell \Delta y, \mathcal{W}_{T}\left(\frac{T m}{N}+s_{\ell} \ell\right)\right) .
$$

The rationale behind the approximation in Eq. (14) is that we assume the starting phases to follow a uniform random distribution within one heartbeat, i.e. $\phi_{m} \sim U([0, T))$, which stems from the stochastic nature of data write times to disk and (slight) variability in the heartbeat. Provided sufficient frames are available, such an assumption is reasonable. We have evaluated the accuracy of approximating the true phases by equispaced phases in Section IV-A (specifically, see Fig. 3).

The final step before we can exploit Eq. (15) to reconstruct images of the beating heart is to estimate the heartbeat period $T$, which is unknown. To do so, we assume a candidate period $T^{\prime}$ for $T$ and invert Eq. (13) to obtain $\mathbf{f}_{T^{\prime}}$, a uniformly sampled (without scanning aberration) version of $f$ :

$$
\mathbf{f}_{T^{\prime}}[k, \ell, n]=\mathbf{g}^{\tilde{\sigma}}\left[k, \ell, \mathcal{W}_{N}\left(n-\left(\frac{s_{\ell} N}{T^{\prime}} \ell\right)\right)\right] .
$$

Since the time index in the right-hand-side can be noninteger, we interpolate the data between successive time points. Specifically, we use a cubic B-spline model with periodic boundary conditions [35].

Next, to obtain the estimate $\tilde{T}$ of the underlying period $T$, we minimize the sum of the differences between neighboring rows $\ell$ for reconstructions $\mathbf{f}_{T^{\prime}}$ obtained from different values of the candidate period $T^{\prime}$ :

$\tilde{T}=\underset{T^{\prime}}{\arg \min }\left(\sum_{k=0}^{K-1} \sum_{\ell=0}^{L-2} \sum_{n=0}^{N-1}\left|\mathbf{f}_{T^{\prime}}[k, \ell, n]-\mathbf{f}_{T^{\prime}}[k, \ell+1, n]\right|\right)$.
If the period resulting in the minimal cost is smaller than 0 , then the best estimated permutation is $\tilde{\sigma}^{T}$, and the time points need simply to be mirrored with respect to time.

In practice, we used an implementation (from the Python package SciPy) of the Nelder-Mead simplex method [36] to minimize the expression in Eq. (17).

\section{Scan-aberration compensation via resampling}

Finally, Equation (16), evaluated for the optimal estimate $T^{\prime}=\tilde{T}$, provides our estimate of $\mathbf{f}$ in Eq. (2):

$$
\tilde{\mathbf{f}}=\mathbf{f}_{\tilde{T}} .
$$

\section{EXPERIMENTS}

We have characterized the accuracy of our method and evaluated its applicability in practice, which we detail in the following sub-sections.

\section{A. Expected error due to non-gated, non-uniform sampling}

We first investigated the error that can be expected from the approximation in Eq. (14). Under the assumption that the starting times of the image acquisitions are stochastic and unrelated to the heartbeat (a reasonable assumption given that slight irregularities in the heart-beat are common and that we empirically observed the image write-times to the disk to be stochastic), we compared the error we make by calculating the differences between the actual phases and the regularlyspaced phases that we impose on the ordered sequences during reconstruction. The assumption here is that there is no error in the ordering of the frames (an aspect that we study separately in Section IV-B3). For a given number of frames, we computed the differences between the left-hand side and the right-hand side of Eq. (14), which corresponds to the integral of the absolute difference between realizations of the true phase curves and the linear approximation (see Fig. 3 inset A, B, C). We repeated the experiment, drawing phases from a uniform distribution, i.e. $\phi_{n} \sim U([0, N))$, and for a varying number of frames, $N \in\{10,20,30, \ldots, 300\}$. We observed that the error decreases as the number of frames increases (Fig. 3).

\section{B. Method characterization on synthetic data}

We next characterized the performance of the full reconstruction pipeline with respect to scan speed and number of frames on synthetic data.

1) Simulation framework for synthetic heart and scanning microscope: We simulated a beating heart (as previously described in [25]) and the LSM acquisition process by resampling data generated on a uniform grid. To simulate a beating heart $f(x, y, t)$, we produced synthetic time-series data $\mathbf{f}^{*}[k, \ell, n]$, where $k=0, \ldots, 255, L=0, \ldots, 255$, and $n=0, \ldots, 2999$. The images featured a periodically contracting ring, with period $T=6 \pi$, which avoided sampling the exact same images over several periods when simulating the scanning artifacts. This sampling protocol represents a nongated acquisition process, where each image is acquired at a different phase. 
To simulate the line scanning acquisition process, we defined the heartbeat-to-frame scanning rate ratio $r=\frac{s_{\ell} L}{T}$ (with units of number of beats per frame (bpf)). With these units, the simulated data can be expressed, based on Eq. (3), as $\mathbf{g}[k, \ell, n]=\mathbf{f}^{*}\left[k, \ell, t+\frac{\ell r T}{L}+n r T\right]$, where $t \in U[0, T)$ is a random starting point in the period. At constant acquisition rate, $r$ increases when the heartbeat increases, while at a constant heart rate, an increase in $r$ corresponds to a decrease in the scanning speed. This simulation allowed us to investigate the influence of two imaging parameters: the number of frames $N$ and the heartbeat-to-frame scanning rate ratio $r$. We first fixed the heartbeat-to-frame scanning rate ratio $r$ and varied $N$. Then, we fixed the final number of frames $N$ and varied $r$. See Supplementary Movie 2 as an illustration.

2) Noise simulation: To make our simulation more realistic, we considered each pixel $i$ to be a realization of a Poisson process whose parameter we set to the noise-free pixel value $f_{i}$ (in the range 0 to 1 ) times a global $\lambda$ level (proportional to the product of photon emission rate and detector dwell time), such that the probability of measuring $k$ photons on a particular pixel was $p_{i}(k)=\mathrm{e}^{-\lambda f_{i}} \frac{\left(\lambda f_{i}\right)^{k}}{k !}$. We simulated data with three global parameters, $\lambda=1, \lambda=10$, and $\lambda=100$, which on a pixel with noise-less intensity $f_{i}=1$, leads to measuring (on average) 1,10 , and 100 photons, respectively.

3) Evaluation of sorting accuracy: To characterize the accuracy of the frame-sorting method that uses the TSP solver with the image-based distance, we carried out the following experiments. We averaged and downscaled the simulated data by a factor 4 , then sorted the downscaled data according to the solution of the TSP solver. We next inspected if the known ground truth phases attached to each frame were in proper sequence. Specifically, to quantify the sorting accuracy we defined the score:

$$
\mathcal{E}[n]=\left\{\begin{aligned}
1 & \phi_{\tilde{\sigma}(n)}<\phi_{\tilde{\sigma}\left(\langle n+1\rangle_{N}\right)} \\
1 & \phi_{\tilde{\sigma}(n)}=\phi_{\max } \text { and } \phi_{\tilde{\sigma}\left(\langle n+1\rangle_{N}\right)}=\phi_{\min } \\
-1 & \text { otherwise, }
\end{aligned}\right.
$$

where $\phi_{\min }$ and $\phi_{\max }$ are the minimal and maximal ground truth phases, respectively and $\langle n\rangle_{N}=n \bmod N$. When correctly sorted, the phases should be in a strictly increasing or decreasing order, with a single jump between the minimal and maximal phases $\phi_{\min }$ and $\phi_{\max }$ and we defined the overall sequence sorting score as follows:

$$
\overline{\mathcal{E}}=\left|\frac{1}{N} \sum_{n=0}^{N-1} \mathcal{E}[n]\right|,
$$

with $0 \leq \overline{\mathcal{E}} \leq 1$, where a low score expresses poor sorting and a high score a correct sorting (the score does not penalize an inverted overall direction of the sorting). We additionally quantified this sorting score by measuring the error magnitude, i.e. the frame positional error as a percentage of the number of frames $N$.

First we studied the influence of the number of frames on the sorting accuracy by varying $N \in\{10,20, \ldots, 300\}$, keeping the heartbeat-to-frame scanning rate ratio fixed to $r=2 \sqrt{2} \mathrm{bpf}$ (Fig. 4). In the noiseless case, the average sorting score does not vary much as the number of frames increases. Although the

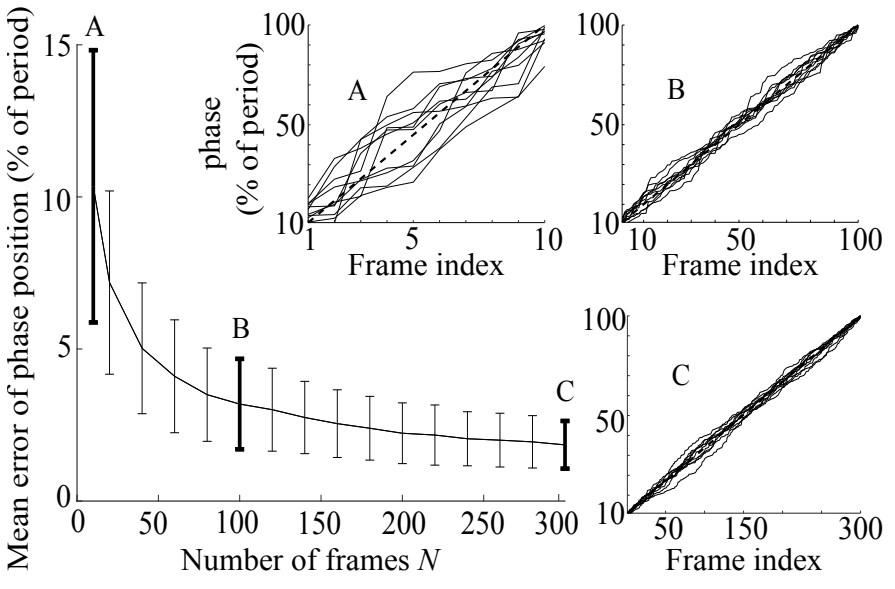

Fig. 3. Accuracy of the equispaced phases approximation. Mean distance between true and equispaced (approximated) phases assuming phases follow a uniform random distribution over the interval of a cardiac period and that sorting is perfect. Insets $\mathrm{A}, \mathrm{B}$, and $\mathrm{C}$ show the true phase as a function of the frame and the deviation from the equispaced phase assignment (diagonal) when the number of frames is 10,100 , and 300 , respectively (10 realizations shown in each case).

sorting score decreases with higher noise levels $(\lambda=1, \lambda=$ 10) the relative phase error induced remains low (Fig. 4(b)).

Next, we set $N=100$ and varied the heartbeat-to-frame scanning rate ratio $r \in\{0.5 \sqrt{2}$ bpf, $\sqrt{2}$ bpf, $\ldots, 9.5 \sqrt{2}$ bpf $\}$ Fig. 4(a). The quality of the reconstruction decreases as the number of heartbeats per frame $r$ increases. This indicates that while a slow scanning speed ( $r$ large) may be acceptable, the reliability of the sorting method is best when the scanning speed is fast ( $r$ is small).

4) Characterization of the accuracy of the period determination method: In order to characterize the accuracy with which we can estimate the period of the heartbeat, we calculated the normalized difference between the estimated period $\tilde{T}$ and the ground truth period $T$ :

$$
\mathcal{E}_{\tilde{T}}=\frac{|T-\tilde{T}|}{T} .
$$

Similarly to Section IV-B3, we considered first a fixed scan rate ratio $r$ and varied $N$, then we fixed $N$ and varied $r$ (Figs 4(c) and 4(d), respectively). We observed that increasing the number of images $N$ (Fig. 4(c)) increases the accuracy of the heartbeat period estimation with a lower variability as we repeated the experiment with multiple realizations of the starting phases. Decreasing the scanning speed (increasing $r$ ) improves the accuracy of the heartbeat period estimation, with less variability as the experiment is repeated over multiple realization of the random starting phases of the frames. We observe that the estimation is robust to photon-count parameter as low as $\lambda=10$, with the method becoming unreliable for $\lambda=1$.

5) Evaluation of the overall accuracy of the proposed reconstruction method: To quantify the end-to-end quality of our entire reconstruction procedure we calculated the intersection over union (IOU [37]) index between ground truth and reconstructed images. The IOU index gives a quantification of the image overlap, or in our case a quantification of the overall 

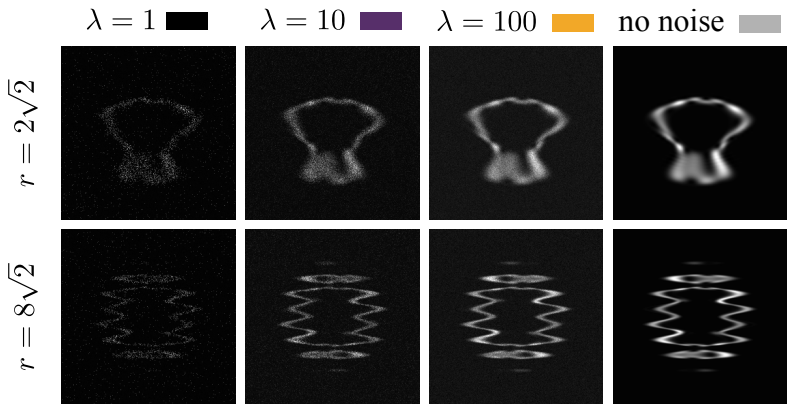

(a)

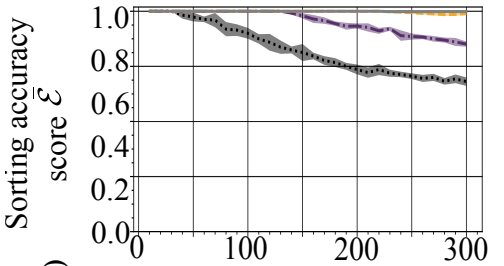

(b)

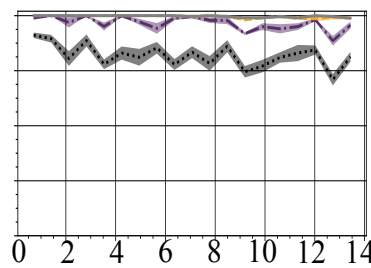

(c)

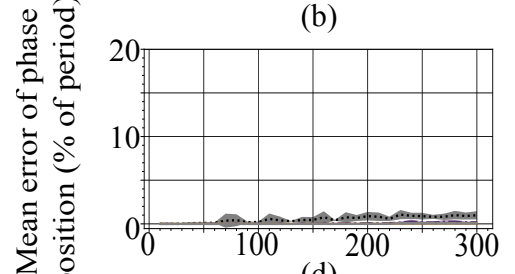

(d)

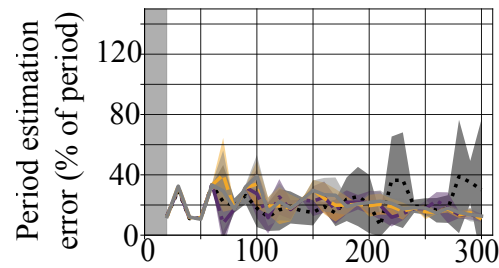

Number of frames $N$

(f)

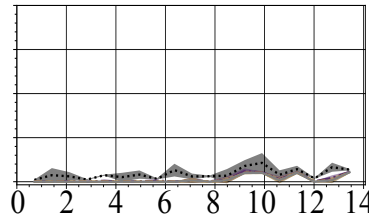

(e)

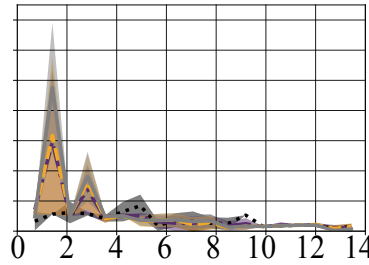

Heartbeat-to-frame scanning rate ratio $r(\mathrm{bpf})$

(g)

Fig. 4. Accuracy of the frame sorting and period estimation procedures. (a) Simulated data with decreasing levels of Poisson noise (Section IV-B2), and without noise. The upper row shows data with a heartbeat-to-scanning rate ratio $r$ of $2 \sqrt{2}$, and the lower row of $8 \sqrt{2}$. (b)-(c) Accuracy of the frame sorting procedure (see Eq. (20)) based on synthetic image data when (b) $r=2 \sqrt{2}$ bpf is fixed while the number of frames $N$ varies and (c) $N=100$ is fixed and $r$ varies. The lines represent the mean over 10 realizations of the simulated movies, and the shadings the standard deviation. Darker colors indicate a higher level of noise, i.e. a lower value of $\lambda$. (d)-(e) Phase position error as a percentage of period when (d) $r=2 \sqrt{2} \mathrm{bpf}$ is fixed while $N$ varies and (f) $N=100$ frames is fixed and $r$ varies. (f) $-(\mathrm{g})$ Positional error as a percentage of number of frames $N$ (f) $r=2 \sqrt{2}$ bpf is fixed while $N$ varies and (g) $N=100$ frames is fixed and $r$ varies. (f) The vertical shading for $N \in[0,19]$ indicate low reliability.

reconstruction quality. As the IOU requires binary images, we applied the reconstruction parameters (phase ordering and period estimation) to the noise-free data equivalent to our noisy datasets. We used the mean of the images as a threshold.

For medium to high photon counts $(\lambda=10,100$, no noise), increasing $N$ improves the overall reconstruction quality (Figs. 5(a) and 5(c)). In high-noise conditions $(\lambda=1)$ the reconstruction quality becomes variable as $N$ increases, in agreement with the worsening sorting and phase estimation performance at these low light conditions (Fig. 5). As the scan rate ratio $r$ is increased, the overall reconstruction quality decreases

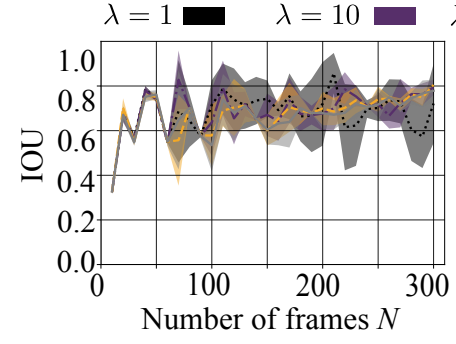

(a) $\lambda=100$

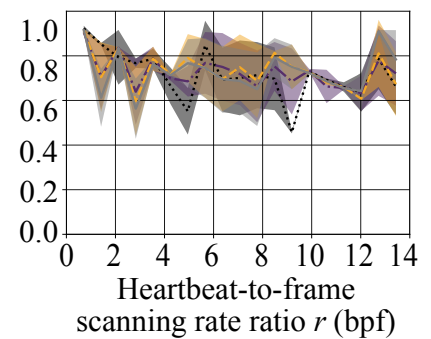

(b)

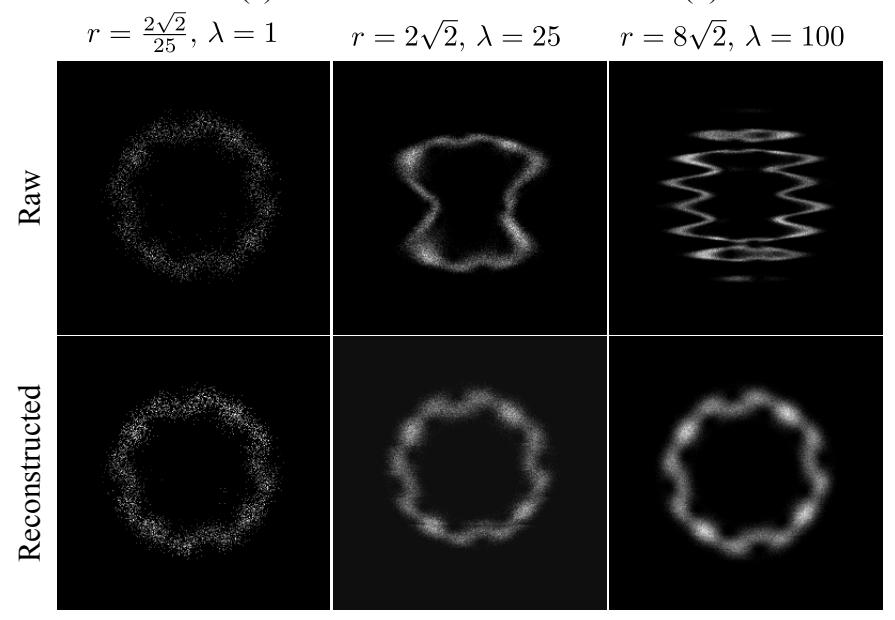

(c)

Fig. 5. The image reconstruction quality (IOU) on simulated data compared to ground truth (a)-(b). Reconstruction quality when: (a) the heartbeat-toframe scanning rate ratio $r=2 \sqrt{2}$ bpf is fixed while the number of frames $N$ varies and (b) the number of frames $N=100$ is fixed and $r$ varies. Each line corresponds to the mean of one level of noise, over 10 realizations, and the shadings the standard deviation. (c) High acquisition speed data, $r=\frac{2 \sqrt{2}}{25}$, $\lambda=1$ versus lower speeds acquisition, $r=2 \sqrt{2}, \lambda=25$, and $r=8 \sqrt{2}$, $\lambda=100$, from left to right. The upper row is the acquired data, the lower row is the reconstructions. See also Supplementary Movie 11.

slightly (Figs. 5(b) and 5(d)). However, this result assumes that it is possible to keep the photon count constant as the scanning speed is increased. In practice, a slower scanning rate, leads to longer dwell times and proportionally larger photon counts (at constant excitation intensity). To illustrate the benefits of using slower scan rates (higher $r$ ), we compared the reconstructions obtained by simultaneously slowing the scanning rate $(r=2 \sqrt{2} / 25,2 \sqrt{2}, 8 \sqrt{2})$ and proportionally increasing the photon counts $(\lambda=1,25,100$, Fig.5(c) and Supplementary Movie 10). Reconstructions show that the shape of the synthetic heart is recovered in all cases, but that the main benefit of a longer dwell-time-the associated higher photon count-can be reaped by compensating the accompanying scanning artefacts with our proposed method.

6) Evaluation of the reconstruction time: We timed the reconstructions on a Debian GNU/Linux 9.9 Operating System, with an Intel Core i7-5930K 3.50GHz CPU, and 32GB RAM (see Fig. 6). Within the probed range of frames, we observed that the reconstruction time increases linearly with the number of frames and stays constant as the heartbeat-toframe scanning rate ratio increases. As shown in a detailed example in Section IV-C, the resampling step is the most time consuming, and scales linearly with the data size. The sorting 


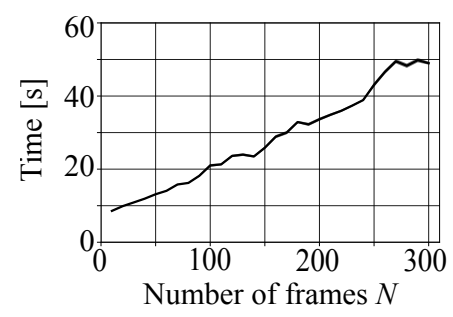

(a)

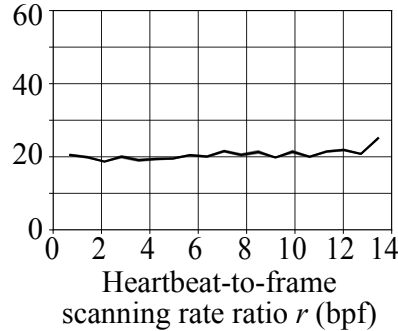

(b)

Fig. 6. The computation reconstruction time increases with (a) the number of frames $N$, rather than (b) the number of beats per frame $r$.

step scales exponentially with the data size as mentioned in Section IV-A but contributes less to the overall computational cost.

\section{Reconstruction of a beating heart sequence from experi- mental measurements}

1) Methods: In order to evaluate the potential of our method for biological applications using standard LSMs, we acquired data in live samples. We bred transgenic zebrafish (Tg(myl7:GFP) [38] and $\operatorname{Tg}$ (myl7:membranemCherry) [39]), which express fluorescent proteins in the heart (green and red fluorescent protein, respectively, in the muscle cells of the entire heart) according to standard procedures [40]. All procedures were previously authorized by the Veterinary Office of the Canton of Bern, Switzerland, for conformity. We grew the zebrafish embryos in E3 medium and added PTU $(0.003 \%$ 1-phenyl-2-thiourea) to avoid pigmentation when the embryos reached an age of 24 hours post fertilization (hpf). At an age of $36 \mathrm{hpf}$, we removed the chorion surrounding the embryos with forceps and anesthetized the embryos with Tricaine at $0.08 \mathrm{mg} / \mathrm{ml}, \mathrm{pH}$ 7. We embedded the embryos with the ventral side down on a glass bottom dish in $1 \%$ low melting agarose (Promega). We imaged the heart on a Zeiss LSM880 inverted confocal microscope with an LD C-Apochromat $40 \times / 1.1 \mathrm{NA}$ water immersion objective lens.

2) Qualitative evaluation on multiple samples: We imaged the hearts of $\operatorname{Tg}($ myl7:GFP) (Fig. 8, Supplementary Movie 3 ) in single direction scanning mode and the hearts of Tg(myl7:membranemCherry) in both single (Fig. 9, Supplementary Movies 5-9) and bi-direction (Fig. 7, Supplementary Movie 4) scanning modes. The reconstructions allow recovering the sequential beating of the heart, with atrium and ventricle beating distinctively one after the other (Fig. 7 and Supplementary Movie 4).

In order to evaluate the practical potential of our method on a multi-channel data set, we further imaged the transgenic $\operatorname{Tg}$ (myl7:membranemCherry)) zebrafish and simultaneously collected fluorescence emission and transmitted light. The duration to scan a full frame of $1024 \times 1024$ pixels was 1270 $\mathrm{ms} /$ frame, with a corresponding duration to scan a line of $s_{\ell}=1.24 \mathrm{~ms}$. We acquired $N=100$ images. Based on those numbers and our method, we estimated the period to be $\tilde{T}=0.33$ seconds, which corresponds to $\frac{1}{\tilde{T}}=3.0$ heartbeats per second. The reconstructed sequence of 100 images, which

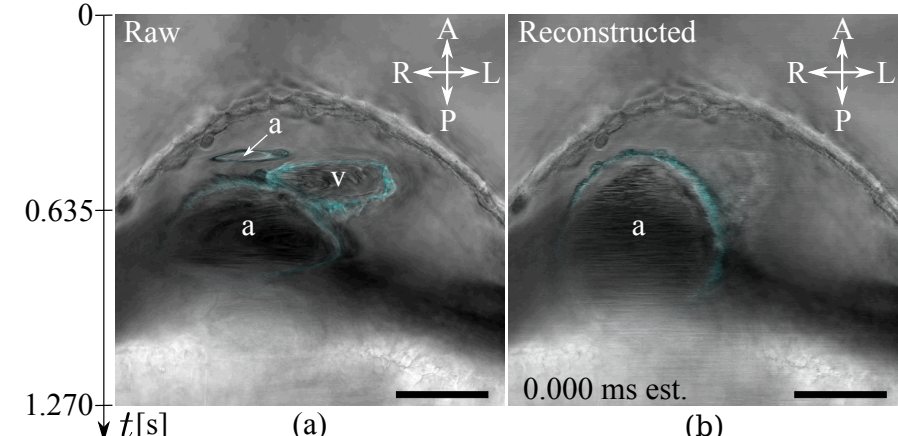

Fig. 7. This dataset features both chambers of the beating heart. The images were acquired in transmission (gray) and fluorescence (cyan) simultaneously. (a) Raw frame: several heartbeats are co-mingled. In particular the atrium (marked by 'a') a single chamber of the heart, appears as two separate chambers. Both the atrium and the ventricle (marked by ' $v$ ') show deformations due to the slow acquisition. (b) Reconstructed heart. The atrium is properly shaped. The contracted ventricle is out of the field of view in this frame (full sequence in Supplementary Movie 4). A: anterior, P: posterior, L: left, R: right. Scale bar is $50 \mu \mathrm{m}$. Additional reconstructions can be seen in Supplementary Movies 7-9

covers one heartbeat, therefore has a virtual frame rate of 300 $\mathrm{Hz}$. For this dataset, the whole reconstruction took 1 minute and 9 seconds: it took 3.4 seconds to load the data (in CZI format); calculating the distance table $\mathbf{D}$, finding the TSP solution, and sorting the data took 3.1 seconds; estimating the period took 2.1 seconds; resampling took 41.3 seconds; finally, saving the data both to MP4 and OME formats took 21.7 seconds. This reconstruction was performed on a Debian GNU/Linux 9.9 Operating System, with an Intel(R) Core(TM) i7-5930K CPU 3.50GHz, and 32GB RAM.

\section{Reconstruction independence on scanning direction}

To verify that the reconstructions were not biased by the mounting orientation with respect to the scanning direction, we acquired two movies of the $\mathrm{Tg}$ (myl7:GFP) zebrafish heart with the scan-field rotated by 0 and 90 degrees (see Figs. 8(a) and $8(\mathrm{~b})$, respectively). We super-imposed both reconstructed datasets (Fig. 8(c)), in the green channel for one scan-field orientation and in the purple channel for the 90-degree rotated orientation. Discrepancies in the reconstructions are visible whenever the signal is not white, which occurs predominantly in the fastest phases of the cardiac cycle as can be seen in Supplementary Movie 3.

\section{E. Comparison to state-of-the-art fast microscopes}

In order to compare our method with the fastest (commercially) available microscopes, we carried out the following experiments. First, we took advantage of the fact that the LSM we used (Zeiss LSM880) was equipped with an Airyscan module, which permits scanning at high speed and allowed us to do a direct comparison (on the same sample) with standard LSM (Fig. 9(d)-(f)).

In addition, we acquired images with a Leica TCS SP8 microscope in both LSM and light sheet mode. To do so, we prepared a U-shaped capillary on plasticine in a glass bottom MatTek dish. Subsequently, we immersed a zebrafish 


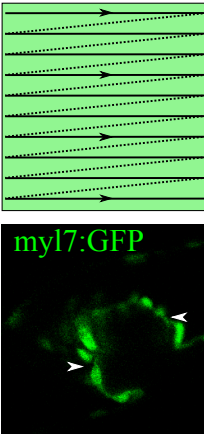

(a)

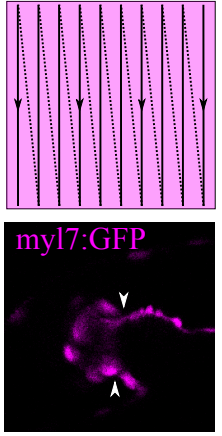

(b)

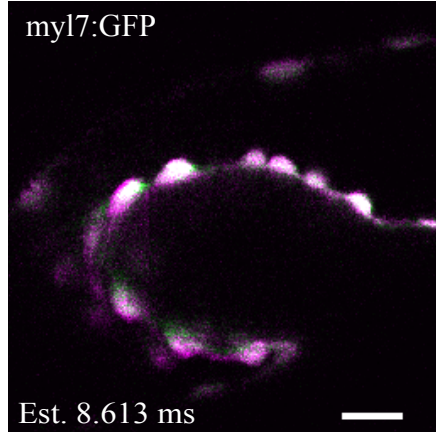

(c)
Fig. 8. Reconstruction is independent of scan field orientation. (a) Top: single-direction, row-first scanning. Bottom: Raw scan frame acquired prior to reconstruction has artifacts: arrows indicate pinching of the membrane that is due to presence of multiple phases of the heartbeat in one frame (scanning artifact). (b) Top: scan-field is rotated by 90 degrees. Bottom: Similar scanning artifacts are visible (arrows). (c) Composite frame of the reconstructed movies obtained from the original and rotated scan-field data, in the green and purple channels, respectively. Predominantly white areas show consistent reconstruction and independence from scan direction. See also Supplementary Movie 3. Scale bar is $20 \mu \mathrm{m}$.
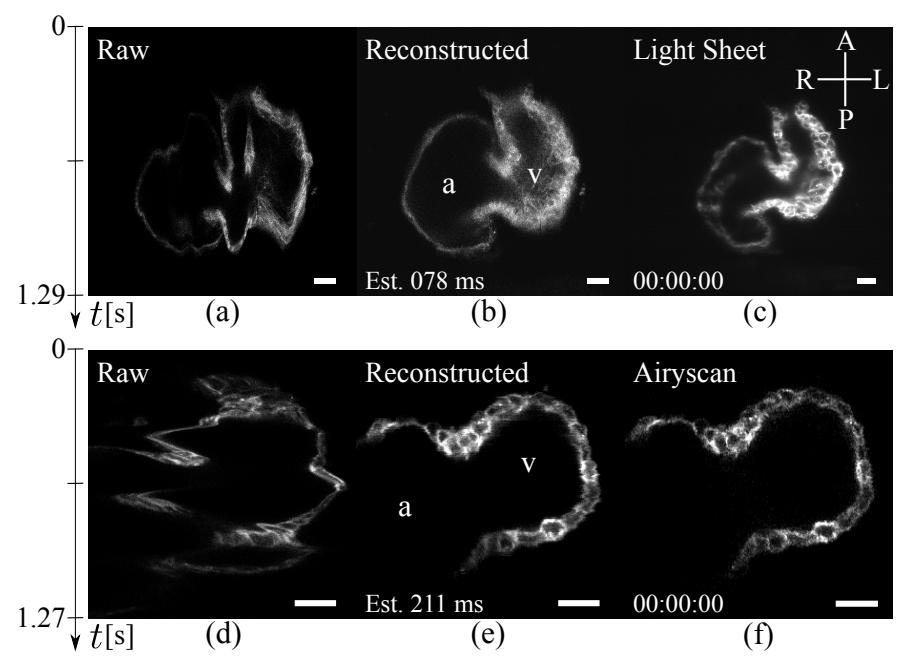

Fig. 9. These datasets feature both chambers of the beating heart. In (a) and (d) several heartbeats are co-mingled. Both the atrium (marked by 'a') and the ventricle (marked by ' $v$ ') show deformations due to the slow confocal microscope acquisition. (b) and (e) show reconstructed hearts. The atrium and ventricle are properly shaped. (full sequences in Supplementary Movies 5 and 6). (c) Data acquired with a light sheet microscope. There are no deformations in this image, as an entire plane in acquired at once. (f) Data acquired with a confocal equipped with an Airyscan. The Airyscan system allows much faster acquisitions, avoiding deformations. A: anterior, P: posterior, L: left, R: right. Scale bar is $20 \mu \mathrm{m}$

larva at $3 \mathrm{dpf}$ in $1 \%$ low melting agarose and mounted it into the capillary, ventral side up. We acquired the images in the light sheet mode. We removed the larva from the capillary and mounted it ventral side down in $1 \%$ low melting agarose in a glass bottom dish. We then acquired the confocal LSM images of the same larva in a similar position (Fig. 9(a)-(c)).

Fig. 9 and Supplementary Movies 5 and 6 show that the reconstructions with our method from data from a slow LSM yield comparable quality reconstruction during the slow phases of the heartbeat, with artifacts remaining when the motion of the heart is at its fastest.

\section{Discussion}

Our method addresses the problem of reconstructing scanning-aberrated images with minimal knowledge of the experimental process, to ensure applicability with a wide range of microscopes (closed commercial or custom-built). Specifically, our method does not require gating the microscope to the heartbeat. Although demonstrated as feasible on custom microscopes, gating requires an additional layer of experimental complexity [41] and is difficult to implement on commercial microscopes, which forbid low-level user intervention during the acquisition process. We also note that although the arbitrariness in the timestamps is not purposeful, as it naturally arises from slow variations in the heartbeat on the sample side or communication lags on the acquisition side, the lack of perfect periodicity is also an advantage as it limits the risk of stratified sampling, which could provide only partial coverage of the cardiac phases.

The first step of our method sorts the frames, which then cover one single period with a higher (virtual) time sampling density. The underlying assumption is that the phase is drawn from a uniform distribution. We further assume that the sorted phases are equally spaced. In [27], we observed that the discrete signal $\mathbf{f}[k, \ell, n]$ (Eq. (2)) had to be asymmetric for the sorting to be successful. Here, since the scanning aberrations encode the movement direction in the images, all images are by construction asymmetric. Our investigation of the uniform distribution with equally-spaced and deterministic samples approximation (Fig. 3), showed that it is less error-inducing as the number of frames $N$ increases. We determined that this error becomes acceptable when $N>100$, as the maximal difference error becomes less than 5\%, meaning that there is less than $5 \%$ difference between the non-uniform time position after sorting and the uniform time position. If the number of frames is lower, $N<100$, then the time difference between two successive sorted frames will have more than 5\% maximal error and lead to important reconstruction artifacts, or even make reconstruction impossible (which we further discuss below). Our choice of using a TSP solver allowed us to find reliable solutions in a very short time (around a second for 100 frames). The sorting error depends on noise, (Fig. 4(a), Fig. 4(b)), but although the number of sorting errors increases, the phase errors remains low (Fig. 4(c), Fig. 4(d)).

The second step estimates the signal period. We observed that acquiring more images leads to a better period estimation and lower variance as we repeat the experiments over multiple realization of the simulated data (Fig. 4(e)). However, very noisy data lead to an increase in period estimation error as $N$ increases, especially over approx. $N=150$ frames. The sorting accuracy for data with $\lambda=1$ is below $75 \%$ when $N>150$. Even if the phase position errors are small (less than $2 \%$ ), the accumulation of errors leads to an increasing error in period estimation; for $\lambda=10$ the estimated period error is $30 \%$ at most, and for $\lambda=100$ is around $10 \%$. For fewer than $N=100$ frames, the standard deviation of the error increases and reconstructions are less reliable for all levels of noise. As $r$ increases, the mean and standard deviation drop very quickly (Fig. 4(f)). The error on the estimated period decreases 
from around $20-40 \%$, when $r=1.8$, to below $5 \%$ for all noise levels, when $r>9$. There is a trade-off to find between the number of frames and the heartbeat-to-framerate ratio. A higher number of heartbeats per frames with a low noise level lead to a better reconstruction than a noisy data acquired with less heartbeats per frame. A high level of noise with a very fast scanner would not need to be reconstructed (4(a) upper left), but the data will have a very low SNR. That could easily be improved with a slower scanning speed (i.e. higher heartbeatto-frame ratio $r$ ), that would allow for a higher SNR with a high reconstruction accuracy.

The overall accuracy (Fig. 5) is better when the number of frames $N$ is high, and the scan rate ratio $r$ is high. The overall accuracy as a function of the scan rate ratio $r$ seems to be mainly limited by the sorting step (Fig. 4(b)), since the estimated period error decreases with higher scan rate ratios (Fig. 4(d)). The overall accuracy is better with a lower noise, and that translates to a slower scanning rate. Data can be acquired with a high scanning speed (Fig. 5(c), upperleft image), without any visible scanning aberration in the data. In that case, the data do not need to be reconstructed to compensate for scanning artifacts, but they are very noisy. When slowing the scanning speed, the data becomes scanningaberrated but also less noisy ((Fig. 5(c) upper-middle and right). The reconstruction quality increases with longer integration times (Fig. 4(b)-(g), Supplementary Movie 10).

Coarse image structures appear sufficient to provide the necessary information to sort and estimate the heartbeat period and we can therefore average and downsize raw images for processing, which both reduces image noise (improving the reconstruction accuracy) and computation time. In practice we used a downsizing factor of 4 for a minimal image size of $64 \times 64$ pixels, which is sufficient for a good reconstruction, but for raw images whose size is less than $256 \times 256$, reconstruction becomes less reliable.

Our method assumes that the cardiac motion is both spatially and temporally cyclic and deviations from this assumption lead to reconstruction artifacts. While our method could be used in perturbation experiments that maintain a regular heartbeat (e.g. perturbations that slow or accelerate the heartbeat yet maintaining it stationary [42], [43]) it would not be applicable to study the effect of more general perturbations or diseased hearts that exhibit severe arrhythmia.

\section{CONCLUSION}

In this paper we proposed a method to reconstruct image series of the beating heart from scanning-aberrated microscopy images. The method relies on a fast TSP solver to sort images according to a minimal frame-to-frame image difference and we use a spatial difference criterion to compensate for scanning aberrations.

We characterized the method on synthetic data, and showed that data acquired at a frame rate up to 4 heartbeats per period (equivalent to a duration to scan a full image of about a second per frame in the case of the beating heart of a zebrafish larva) for 100 frames allows over $75 \%$ reconstruction accuracy (IOU, noise up to $\lambda=10$ ). This accuracy can be improved by increasing the number of collected frames (at the cost of a longer overall image acquisition time). We showed that our method allows reconstructing data with a virtual framerate of up to $300 \mathrm{~Hz}$. We demonstrated that the method is applicable in practice on data from a standard confocal LSM, providing reconstruction quality similar to that of state-of-theart fast microscopes in the slower phases of the heartbeat (with remaining artifacts in the faster phases). We foresee that this reconstruction method could be applied to other point- or line-scanning microscopy modalities (in particular, for multi-photon imaging, where alternatives to point scanning are even fewer). Our simulations provide guidelines on the critical parameters (number of frames and scanning speed) to be adjusted and an experimental procedure to verify the validity of a reconstruction, by varying the sample orientation with respect to the scanning direction. Future work could include extensions for sub-pixel estimation of frame positions and automatic detections of arrhythmias, where our method would not be applicable.

\section{ACKNOWLEDGMENTS}

This work is supported by a project funded jointly by the Agence nationale de la recherche (ANR) and the Swiss National Science Foundation (SNSF), Grant 310030E-164245 "liveheart: The cellular basis of cardiac development revealed by live imaging," and the Swiss National Science Foundation Grant 206021_164022 "Platform for Reproducible Acquisition, Processing, and Sharing of Dynamic, Multi-Modal Data." Thanks to Xavier Langa and Ronja Baal for fish husbandry. This work used Microscopes from the MIC-Bern Facility.

\section{REFERENCES}

[1] A. Zhang, Q. Zhang, C.-L. Chen, and R. K. Wang, "Methods and algorithms for optical coherence tomography-based angiography: a review and comparison," J. Biomed. Opt., vol. 20, no. 10, pp. 1-11, Oct. 2015.

[2] M. W. Jenkins, O. Q. Chughtai, A. N. Basavanhally, M. Watanabe, and A. M. Rollins, "In vivo gated 4D imaging of the embryonic heart using optical coherence tomography," J. Biomed. Opt., vol. 12, no. 3, p. 030505 , May 2007.

[3] A. Liu, R. Wang, K. Thornburg, and S. Rugonyi, "Efficient postacquisition synchronization of 4-D nongated cardiac images obtained from optical coherence tomography: application to 4-D reconstruction of the chick embryonic heart," J. Biomed. Opt., vol. 14, no. 4, pp. 1-11, Jul. 2009.

[4] I. V. Larina, K. V. Larin, M. E. Dickinson, and M. Liebling, "Sequential Turning Acquisition and Reconstruction (STAR) method for four-dimensional imaging of cyclically moving structures," Biomed. Opt. Express, vol. 3, no. 3, p. 650, Mar. 2012.

[5] J. Rietdorf and E. H. K. Stelzer, "Special optical elements," in Handbook of Biological Confocal Microscopy, 3rd ed., J. B. Pawley, Ed. Springer Science \& Business Media, 2006, ch. 3, pp. 52-54.

[6] P. Gonnord and R. Varma, "Imaging distributions of molecules in motile cells by high-speed confocal microscopy," in Fluorescent Methods to Study Biological Membranes, ser. Springer Series on Fluorescence, Y. Mely and G. Duportail, Eds. Springer Science \& Business Media, 2013, vol. 13 , ch. 2.2 , pp. $369-370$.

[7] J. Huff, A. Bergter, J. Birkenbeil, I. Kleppe, and R. Engelmann, "The new 2D superresolution mode for ZEISS Airyscan," Nat. Methods, vol. 14, no. 12, pp. iv-vi, Nov. 2017.

[8] D. Toomre and J. B. Pawley, "Disk-scanning confocal microscopy," in Handbook of Biological Confocal Microscopy, 3rd ed., J. B. Pawley, Ed. Springer Science \& Business Media, 2006, ch. 10, pp. 231-232.

[9] M. Liebling, A. S. Forouhar, R. Wolleschensky, B. Zimmermann, R. Ankerhold, S. E. Fraser, M. Gharib, and M. E. Dickinson, "Rapid three-dimensional imaging and analysis of the beating embryonic heart reveals functional changes during development," Dev. Dyn., vol. 235, no. 11 , pp. 2940-2948, Nov. 2006 
[10] P. Lam, R. S. Fischer, W. D. Shin, C. M. Waterman, and A. Huttenlocher, Spinning disk confocal imaging of neutrophil migration in zebrafish, ser. Methods in Molecular Biology. Humana Press, 2014, vol. 1124, ch. 14, pp. 219-233.

[11] S. Inoué and T. Inoué, Direct-View High-Speed Confocal Scanner: The CSU-10, ser. Methods in Cell Biology. Elsevier, 2002, vol. 70, pp. $87-127$.

[12] J. Huisken and D. Y. R. Stainier, "Selective plane illumination microscopy techniques in developmental biology," Development, vol. 136, no. 12, pp. 1963-1975, Jun. 2009.

[13] M. Mickoleit, B. Schmid, M. Weber, F. O. Fahrbach, S. Hombach, S. Reischauer, and J. Huisken, "High-resolution reconstruction of the beating zebrafish heart," Nat. Methods, vol. 11, no. 9, pp. 919-922, Sep. 2014.

[14] J. M. Taylor, J. M. Girkin, and G. D. Love, "High-resolution 3D optical microscopy inside the beating zebrafish heart using prospective optical gating," Biomed. Opt. Express, vol. 3, no. 12, p. 3043, Dec. 2012.

[15] P. Mahou, J. Vermot, E. Beaurepaire, and W. Supatto, "Multicolor twophoton light-sheet microscopy," Nat. Methods, vol. 11, no. 6, pp. 600601, Jun. 2014.

[16] P. Pitrone, J. Schindelin, L. Stuyvenberg, S. Preibisch, M. Weber, K. Eliceiri, J. Huisken, and P. Tomancak, "OpenSPIM: An open-access light-sheet microscopy platform," Nat. Methods, vol. 10, pp. 598-599, 2013.

[17] E. J. Gualda, T. Vale, P. Almada, J. A. Feijó, G. G. Martins, and N. Moreno, "OpenSpinMicroscopy: an open-source integrated microscopy platform," Nat. Methods, vol. 10, p. 599, 2013.

[18] J. Huff, "The Airyscan detector from ZEISS: confocal imaging with improved signal-to-noise ratio and super-resolution," Nat. Methods, vol. 12, p. 1205, 2015.

[19] Q. Zhang and R. Pless, Segmenting cardiopulmonary images using manifold learning with level sets, ser. Lecture Notes in Computer Science. Berlin, Germany: Springer, 2005, vol. 3765 , ch. 48, pp. 479488.

[20] J. B. Tenenbaum, V. de Silva, and J. C. Langford, "A global geometric framework for nonlinear dimensionality reduction," Science, vol. 290 pp. 2319-2323, Dec. 2000.

[21] P. Kellman, C. Chefd'hotel, C. H. Lorenz, C. Mancini, A. E. Arai, and E. R. McVeigh, "High spatial and temporal resolution cardiac cine MRI from retrospective reconstruction of data acquired in real time using motion correction and resorting," Magn. Reson. Med., vol. 62, no. 6, pp. 1557-1564, Dec. 2009.

[22] L. Feng, L. Axel, H. Chandarana, K. T. Block, D. K. Sodickson, and R. Otazo, "XD-GRASP: Golden-angle radial MRI with reconstruction of extra motion-state dimensions using compressed sensing," Magn. Reson. Med., vol. 75, no. 2, pp. 775-788, Feb. 2016.

[23] C. M. Happel, J. Thommes, L. Thrane, J. Mnner, T. Ortmaier, B. Heimann, and T. M. Yelbuz, "Rotationally acquired four-dimensional optical coherence tomography of embryonic chick hearts using retrospective gating on the common central A-scan," J. Biomed. Opt., vol. 16 , no. 9, pp. 1-7, Sep. 2011.

[24] M. M. Dworetsky, "A period-finding method for sparse randomly spaced observations or "How long is a piece of string?"," Mon. Not. R. Astron. Soc., vol. 203, no. 4, pp. 917-924, Aug. 1983.

[25] M. Liebling, A. S. Forouhar, M. Gharib, S. E. Fraser, and M. E. Dickinson, "Four-dimensional cardiac imaging in living embryos via postacquisition synchronization of nongated slice sequences," J. Biomed. Opt., vol. 10, no. 5, pp. 1-10, 2005.

[26] C. J. Tralie and M. Berger, "Topological Eulerian synthesis of slow motion periodic videos," in 2018 25th IEEE Int. Conf. Image Proces. (ICIP). IEEE, Oct. 2018, pp. 3573-3577.

[27] O. Mariani, K. G. Chan, A. Ernst, N. Mercader, and M. Liebling, "Virtual high-framerate microscopy of the beating heart via sorting of still images," in 2019 IEEE 16th Inter. Symp. on Biomed. Imaging, (ISBI 2019), Apr. 2019, pp. 312-315.

[28] P. Hanslovsky, J. Bogovic, and S. Saalfeld, "Image-based correction of continuous and discontinuous non-planar axial distortion in serial section microscopy," Bioinformatics, vol. 33, no. 9, pp. 1379-1386, May 2017.

[29] G. De Haan and E. B. Bellers, "Deinterlacing-an overview," Proc. IEEE, vol. 86, no. 9, pp. 1839-1857, Sep. 1998.

[30] S. Lee, C. Vinegoni, M. Sebas, and R. Weissleder, "Automated motion artifact removal for intravital microscopy, without a priori information," Sci. Rep., vol. 4, Mar. 2014.

[31] W. Cook, "Concorde Home." [Online]. Available: http://www.math. uwaterloo.ca/tsp/concorde.html
[32] D. Applegate, W. Cook, S. Dash, and M. Mevenkamp, "QSopt linear programming solver." [Online]. Available: http://www.math.uwaterloo. $\mathrm{ca} / \sim$ bico/qsopt/index.html

[33] D. L. Applegate, R. E. Bixby, V. Chvatal, and W. J. Cook, The Traveling Salesman Problem: A Computational Study, ser. Princeton Series in Applied Mathematics. Princeton, NJ, USA: Princeton University Press, 2007.

[34] H. H. Hoos and T. Stützle, "On the empirical time complexity of finding optimal solutions vs proving optimality for Euclidean TSP instances," Optim. Lett., vol. 9, no. 6, pp. 1247-1254, Aug. 2015.

[35] M. Unser, "Splines: a perfect fit for signal and image processing," IEEE Signal Process. Mag., vol. 16, no. 6, pp. 22-38, Jun. 1999.

[36] J. A. Nelder and R. Mead, "A simplex method for function minimization," Comput. J., vol. 7, no. 4, pp. 308-313, 1965.

[37] P. Jaccard, "Étude de la distribution florale dans une portion des Alpes et du Jura," Bulletin de la société vaudoise des sciences naturelles, vol. 37, pp. 547-579, 1901.

[38] C.-J. Huang, C.-T. Tu, C.-D. Hsiao, F.-J. Hsieh, and H.-J. Tsai, "Germline transmission of a myocardium-specific GFP transgene reveals critical regulatory elements in the cardiac myosin light chain 2 promoter of zebrafish," Dev. Dyn., vol. 228, no. 1, pp. 30-40, Sep. 2003.

[39] S. Rohr, C. Otten, and S. Abdelilah-Seyfried, "Asymmetric involution of the myocardial field drives heart tube formation in zebrafish," Circ. Res., vol. 102, pp. e12-e19, Feb. 2008.

[40] M. Westerfield, The Zebrafish Book: A Guide for the Laboratory Use of Zebrafish, 4th ed. Univ. of Oregon Press, Eugene, 2000.

[41] J. M. Taylor, "Optically gated beating-heart imaging," Front. Physiol., vol. 5, 2014.

[42] J. Ohn and M. Liebling, "In vivo, high-throughput imaging for functional characterization of the embryonic zebrafish heart," in 2011 IEEE International Symposium on Biomedical Imaging: From Nano to Macro, Mar. 2011, pp. 1549-1552.

[43] J. Vermot, A. S. Forouhar, M. Liebling, D. Wu, D. Plummer, M. Gharib, and S. E. Fraser, "Reversing blood flows act through klf2a to ensure normal valvulogenesis in the developing heart," PLOS Biology, vol. 7, no. 11, pp. 1-14, Nov. 2009. 